\title{
Increased anticoagulant activity of thrombin-binding DNA aptamers by nanoscale organization on DNA nanostructures
}

\author{
Abhijit Rangnekar, MSc ${ }^{\mathrm{a}, \mathrm{b}, \mathrm{c}}$, Alex M. Zhang, BS ${ }^{\mathrm{d}}$, Susan Shiyuan Li, BS ${ }^{\mathrm{e}}$, \\ Kristin M. Bompiani, BS ${ }^{\mathrm{f}, \mathrm{g}}$, Majken N. Hansen, $\mathrm{PhD}^{\mathrm{a}}$, Kurt V. Gothelf, $\mathrm{PhD}^{\mathrm{a}}{ }^{\mathrm{b}}$, \\ Bruce A. Sullenger, $\mathrm{PhD}^{\mathrm{f}}$, Thomas H. LaBean, $\mathrm{PhD}^{\mathrm{c}, \mathrm{h}, *}$ \\ ${ }^{a}$ Department of Chemistry and Center for DNA Nanotechnology (CDNA), Aarhus University, Aarhus, Denmark \\ binterdisciplinary Nanoscience Center (iNANO), Aarhus University, Aarhus, Denmark \\ ${ }^{\mathrm{c}}$ Department of Chemistry, Duke University, Durham North Carolina, USA \\ ${ }^{\mathrm{d}}$ Trinity College, Duke University, Durham North Carolina, USA \\ ${ }^{\mathrm{e}}$ Davidson College, Davidson North Carolina, USA \\ ${ }^{\mathrm{f}}$ Department of Surgery, Duke University Medical Center, Durham North Carolina, USA \\ ${ }^{\mathrm{g}}$ University Program in Genetics and Genomics, Duke University, Durham North Carolina, USA \\ ${ }^{\mathrm{h}}$ Department of Computer Science and Department of Biomedical Engineering, Duke University, Durham North Carolina, USA
}

Received 15 June 2011; accepted 10 August 2011

\begin{abstract}
Control over thrombin activity is much desired to regulate blood clotting in surgical and therapeutic situations. Thrombin-binding RNA and DNA aptamers have been used to inhibit thrombin activity and thus the coagulation cascade. Soluble DNA aptamers, as well as two different aptamers tethered by a flexible single-strand linker, have been shown to possess anticoagulant activity. Here, we link multiple aptamers at programmed positions on DNA nanostructures to optimize spacing and orientation of the aptamers and thereby to maximize anticoagulant activity in functional assays. By judicious engineering of the DNA nanostructures, we have created a novel, functional DNA nanostructure, which is a multi-aptamer inhibitor with activity eightfold higher than free aptamer. Reversal of the thrombin inhibition was also achieved by the use of single-stranded DNA antidotes, thus enabling significant control over blood coagulation.
\end{abstract}

From the Clinical Editor: Thrombin inhibition via DNA aptamers has recently become a possibility. In this study, thrombin-binding DNA aptamers were further optimized by nanoscale organization on DNA nanostructures. The authors have created a novel, functional DNA nanostructure, which is a multi-aptamer inhibitor with activity eightfold higher than that of free aptamer. Reversal of thrombin inhibition was also achieved by single-stranded DNA antidotes, enabling significant control over the coagulation pathway.

(C) 2012 Published by Elsevier Inc.

Key words: Thrombin; DNA aptamers; Anticoagulation; DNA nanotechnology; DNA weave-tile

DNA nanotechnology has evolved very rapidly in the past decade. DNA nanostructures have grown both in size and in complexity over time - from the tile-based structures ${ }^{1-5}$ to the origami-based structures. ${ }^{6-8}$ Tile-based nanostructures involve multiple, short, synthetic oligonucleotide strands that selfassemble due to highly specific Watson-Crick pairing between DNA bases. These tiles have proved to be very stable and rigid,

Conflict of interest statement: The authors declare that there are no potential, perceived, or real conflicts of interest.

Financial support was provided by the U.S. National Science Foundation (CCF-0829749, DMR-0706397, CBET-0835794, and OISE-0624012) and by the Danish National Research Foundation.

*Corresponding author: Duke University, Box 90129, Durham, NC 27708, USA.

E-mail address: thomas.labean@duke.edu (T.H. LaBean). mostly owing to the presence of multiple Holliday-junction-like crossovers linking together axially parallel DNA double helices. ${ }^{1,2}$ These tiles have then been used to form a broad range of one- and two-dimensional periodic lattices. ${ }^{2-5,9} \mathrm{~A}$ few finite-sized three-dimensional structures have also been synthesized using tile-based designs. ${ }^{10}$ However, a much higher degree of complexity can be achieved by using origami-based approaches in which a long DNA scaffold strand (typically biosynthesized) is folded into desired two- or three-dimensional shapes by using hundreds of short, synthetic DNA staple strands..$^{6-8}$ These origami structures also attain shape and structural rigidity due to their rigid double-helical domains being tied together by crossovers.

A new building-block design, known as the weave-tile strategy, ${ }^{11}$ was recently reported. (This strategy was independently conceived but not experimentally tested several years ago. ${ }^{12}$ ) This 
A
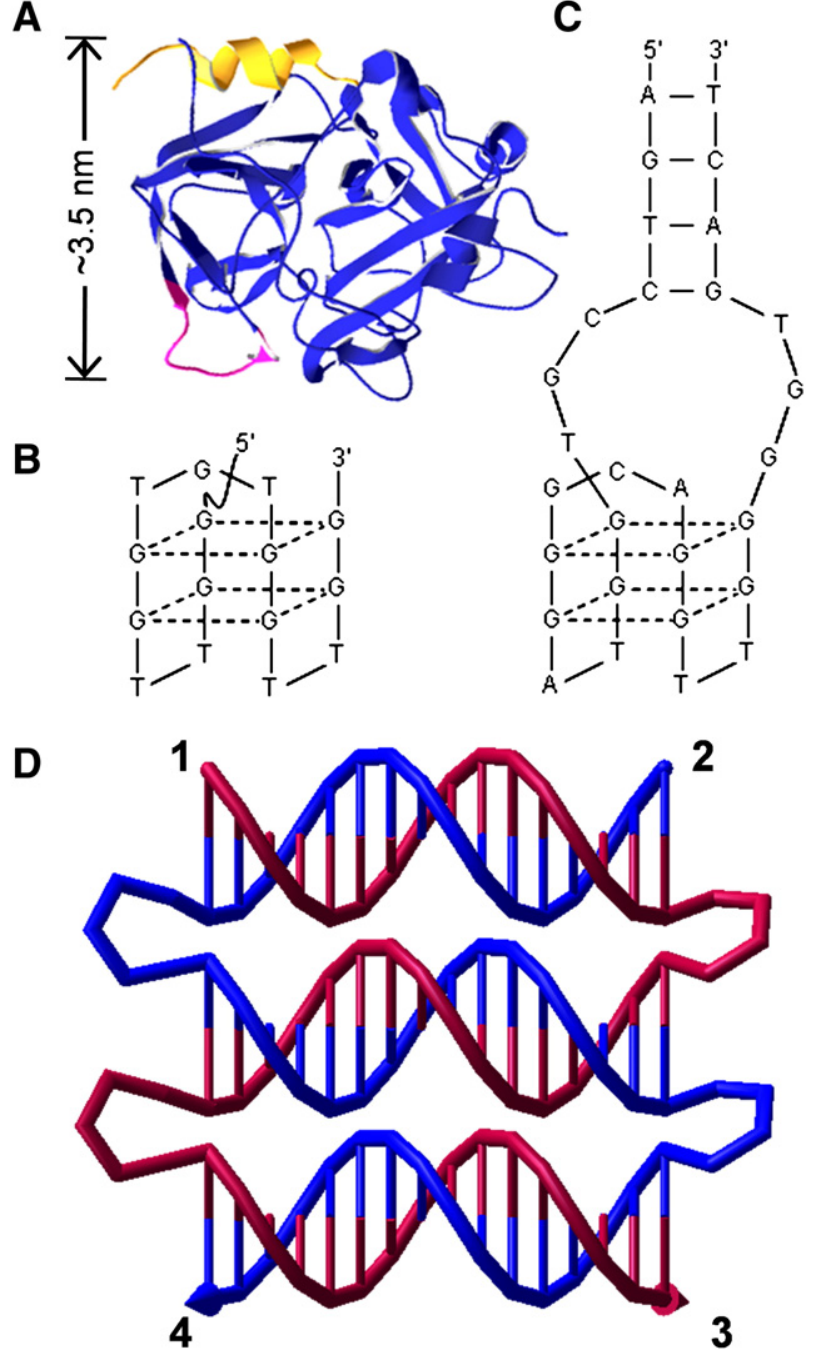

Figure 1. (A) Ribbon representation of thrombin. ${ }^{20}$ Exosite I (magenta) and the exosite II (yellow) are highlighted. (B) Sequence and structure of HD1 aptamer (Aptamer A) which binds to exosite I and inhibits coagulation in plasma clotting assays. ${ }^{20}$ (C) Sequence and structure of 60.29 aptamer (Aptamer B) which has high affinity for binding to the exosite II but low anticoagulant activity. ${ }^{20}$ (D) An example of DNA weave-tile architecture with a representative 3-helix tile (3HT) shown. The four possible locations for the placement of aptamers are also numbered.

strategy requires only 2 DNA strands and avoids the use of crossovers, thereby allowing greater structural flexibility to the DNA nanostructure without compromising stability. The sequences are designed such that they weave back and forth to mimic the strand routing of the origami architectures, thereby forming a weave-like tile with rigid double-helical domains tethered to neighboring domains by flexible single-stranded -TTTT- loops (Figure 1, D). A major goal of DNA nanotechnology is to use DNA nanostructures for precise positioning of proteins, ${ }^{3}$ nanoparticles, ${ }^{13}$ and other functional molecules, such as aptamers ${ }^{14}$ on two-dimensional or three-dimensional templates.

Aptamers are short oligonucleotides (DNA or RNA) that fold into a specific three-dimensional shape; they are isolated by in vitro selection procedures and can display very high specificity and affinity for the target molecules against which they were selected. ${ }^{15-17}$ Thrombin is a multifunctional serine protease and a key component of the blood coagulation cascade. It catalyzes many coagulation-related reactions, including the conversion of soluble fibrinogen to insoluble fibrin and activation of platelets, during blood clot formation. ${ }^{18}$ Regulation of thrombin activity by thrombin-specific inhibitors can play a crucial role during surgical procedures and in the treatment of patients with arterial and venous thromboembolism. ${ }^{19}$ Thrombin inhibition can be achieved by blocking 2 substrate binding sites, known as exosites I and II (Figure 1, $A$ ). Exosite I helps convert fibrinogen to fibrin, whereas exosite II is the heparin binding site and is important for thrombinplatelet interaction. ${ }^{18}$ Several direct thrombin inhibitors that block the enzymatic active site are currently available for clinical use. However, most of them have either harmful side effects or suffer from narrow therapeutic windows. ${ }^{20}$ Nucleic acid aptamers, on the other hand, offer a safe and convenient alternative for anticoagulation because they are nontoxic, their bioavailability can be adjusted through chemical modifications, and they are nonimmunogenic. ${ }^{21}$ Moreover, aptamer anticoagulation activity can be controlled by a specific antidote - an oligonucleotide with a sequence complementary to the aptamer sequence. WatsonCrick base-pairing between the aptamer and antidote disrupts the tertiary structure and thus the function of the aptamer. ${ }^{22}$ Currently, an FIXa anticoagulant aptamer-antidote pair is being developed as an intravenous anticoagulant for clinical use during surgery. ${ }^{23-26}$

Aptamer HD1 (referred to as Aptamer A in this study) binds to exosite I and has significant anticoagulation activity in clinical clotting assays (Figure 1, B). ${ }^{17,20}$ Aptamer 60.29 (referred to as Aptamer B in this study) binds to exosite II and has very high affinity for the thrombin molecule, ${ }^{20}$ but it is not a good inhibitor in plasma clotting assays (Figure 1,C). In the absence of Aptamer B, Aptamer A forms a complex with 2 thrombin molecules, binding to the exosite I of and inactivating one of the thrombin molecules, whereas binding the other thrombin molecule through exosite II which mainly neutralizes the negatively charged backbone of the aptamer. ${ }^{27}$ However, when a copy of Aptamer B is also present, this complex is not formed because of the much higher affinity of Aptamer B for exosite II. ${ }^{20}$ It has been demonstrated that when these 2 aptamers are linked via a singlestranded DNA (ssDNA) linker, the resulting construct has much higher affinity and anticoagulation activity. ${ }^{20}$ However, the ssDNA is a very flexible linker, and because it is structurally disordered, it provides no orientation control and allows for a wide range of possible end-to-end distances. For a bivalent construct, such a high degree of flexibility leads to entropic limitations on effective binding to the target molecule. The affinity and thus anticoagulant activity should improve if a linker platform is used that provides greater conformational control of the aptamers. A previous study demonstrated distance-dependent thrombin binding when Aptamers A and B were appended to rigid, multicrossover DNA tiles ${ }^{14}$; however, anticoagulant activity was not monitored. Such conformationally rigid, polyvalent entities may overcome entropic limitations; however, they can lead to enthalpic limitations. In cases where the linker is too rigid, even small spatial mismatches between the aptamers and their binding sites will result in diminished binding. ${ }^{28}$

Here we demonstrate the use of a DNA weave-tile, which is expected to display intermediate flexibility between ssDNA 
and DNA crossover tiles. We propose that the flexibility of weavetiles enable them to conform more closely and bind more tightly to the thrombin surface, which is known to bear positive charges on surface residues between the exosites. ${ }^{18}$ The ability to conform to the protein surface and electrostatic attractions would be expected to increase the overall affinity between thrombin and the aptamer-tile construct and hence the anticoagulation activity. Moreover, it is possible to control distance between the 2 aptamers as well as the relative orientation of aptamers using the weave-tile as a platform to find a highly effective anticoagulant.

\section{Methods}

\section{DNA strands and tile formation}

Synthetic oligonucleotides were purchased from Integrated DNA Technologies (Coralville, Iowa) in unpurified form and used without further processing. The sequences of all the DNA strands used in this study are provided in the Supplementary Material, available online at http:/www.nanomedjournal.com. Tiles were formed by annealing stoichiometric mixtures of the 2 strands in a buffer consisting of $20 \mathrm{mM}$ HEPES (pH 7.4), $150 \mathrm{mM}$ $\mathrm{NaCl}$ and $2 \mathrm{mM} \mathrm{CaCl}_{2}$. The annealing concentration of each participating strand was $10 \mu \mathrm{M}$. Annealing was performed by incubating the sample in a beaker containing boiling water and letting it stay at the bench top for 90 minutes, followed by incubation of the entire beaker at $4^{\circ} \mathrm{C}$ for 30 minutes.

\section{Weave-tile structures and nomenclature}

The weave-tile architecture can be constructed with any number of helical domains, each $16 \mathrm{bp}$ long (a turn and a half of double helix), connected on the helix ends by ssDNA -TTTT- loops (PAGE gels showing tile formation are shown in Figures S1, S2, and S3 of the Supplementary Material). A weave-tile constructed with three helical domains is thus referenced as 3HT (Figure 1,D). The two 5' and two 3' ends of the oligonucleotide chains, located at the four corners of the tile, can then be utilized to position aptamers. Depending on the design, a portion at the end of the participating ssDNA folds into aptamer and the remaining portion is folded (hybridized) using the other DNA strand to form the double-stranded sections of the weave-tile. In cases where there are two aptamers on the same strand, they both fold individually while annealing and the region flanked by them is folded using the other constituting strand of the weave-tile. For naming the various configurations, available positions are numbered as shown in Figure 1,D and we adopted tile names, such as 3HT-XXXX. Here, depending on the position of the aptamers, X would be replaced by A or B (when the tile has Aptamer $\mathrm{A}$ or Aptamer B at that position), or $\mathrm{n}$ (when there is no aptamer). Aptamer A is connected to the tile via a 7-bp stem and an ssDNA -TT- spacer. ${ }^{11}$ In a control sample with two noncomplementary strands, each bearing a different aptamer, the system is named 3HTNC (no conformation) because no tile is formed.

\section{Nondenaturing polyacrylamide gel electrophoresis}

Gels contained desired amount of acrylamide (19:1, acrylamide:bisacrylamide) in a buffer consisting of $40 \mathrm{mM}$ Tris acetate (pH 8.0), 2 mM EDTA, and $12.5 \mathrm{mM}$ magnesium acetate
$\left(\mathrm{TAE} / \mathrm{Mg}^{++}\right)$. The annealed sample was mixed in equal volume with tracking dye containing $\mathrm{TAE} / \mathrm{Mg}^{++}, 50 \%$ glycerol, and $0.2 \%$ each of Bromophenol Blue and Xylene Cyanol FF. Gels were run on a Hoefer SE-600 electrophoresis unit at $10 \mathrm{~V} / \mathrm{cm}$ and cooled via circulating water bath at $4^{\circ} \mathrm{C}$. The gels were then stained with ethidium bromide and imaged using an AlphaImager (Alpha Innotech, San Leandro, California).

\section{Coagulation assay}

A model ST4 coagulometer (Diagnostica Stago, Parsippany, New Jersey) was used to run an activated partial thromboplastin time (aPTT) assay to test the clotting time for each sample. Reagents include normal pooled human plasma from George King Bio-Medical (Overland Park, Kansas), as well as a $\mathrm{CaCl}_{2}$ solution and TriniClot aPTT reagent from Trinity Biotech (Bray, Wicklow, Ireland). $50 \mu \mathrm{L}$ plasma was activated by adding $50 \mu \mathrm{L}$ TriniClot aPTT reagent and incubated at $37^{\circ} \mathrm{C}$ for 5 minutes, followed by addition of $16.67 \mu \mathrm{L}$ of $10 \mu \mathrm{M}$ sample/control buffer and a further incubation for 5 minutes at $37^{\circ} \mathrm{C}$. Clotting was initiated by addition of $50 \mu \mathrm{L} \mathrm{CaCl}_{2}$ solution so that the final aptamer concentration was $1 \mu \mathrm{M}$. All the tests presented here were conducted at the same final concentration of Aptamer A, unless otherwise stated. The machine automatically measured the time until clot formation after the addition of $\mathrm{CaCl}_{2}$ and the clotting times were recorded and compared. Each test was conducted in triplicate to ensure reliable results. All data presented in this study (except data for the timecourse study) are the averages of tests conducted on at least 2 different days. The data for each sample are represented as the average of all the clotting times for the sample obtained over all the conducted tests. The error bars represent the standard error of the mean (SEM).

\section{Anticoagulation reversal using antidotes}

For the antidote experiments, the plasma was activated and treated with aptamer as described above. The necessary amount of antidote was subsequently added to achieve the desired antidote/aptamer ratio, and the reaction was further incubated for 5 minutes at $37^{\circ} \mathrm{C}$ before $\mathrm{CaCl}_{2}$ addition. The aptamer and antidote concentrations were adjusted for dilution caused because of addition of the antidote. For the antidote time-course study, the time points listed indicate the time between antidote addition and assay initiation with $\mathrm{CaCl}_{2}$.

\section{Residual activity calculation}

The residual activity of aptamer after time $\mathrm{t}$ is calculated against the activity of aptamer after 5 minutes incubation as follows:

$$
\left[\mathrm{T}_{\text {aptamer }, \mathrm{t}}-\mathrm{T}_{\text {control }, \mathrm{t}}\right] /\left[\mathrm{T}_{\text {aptamer }, 5 \mathrm{~min}}-\mathrm{T}_{\text {control }, 5 \mathrm{~min}}\right] \times 100
$$

Here, $\mathrm{T}$ is the clotting time in seconds. Similarly, the residual activity of antidote-aptamer system is calculated as follows:

$\left[1-\left\{\left(\mathrm{T}_{\text {aptamer }}-\mathrm{T}_{\text {aptamer }+ \text { antidote }}\right) /\left(\mathrm{T}_{\text {aptamer }}-\mathrm{T}_{\text {control }}\right)\right\}\right] \times 100$

For free Aptamer A studies, buffer was used as control. However, for 2HT-BnAn tile, 2HT-Bnnn was used as control. 


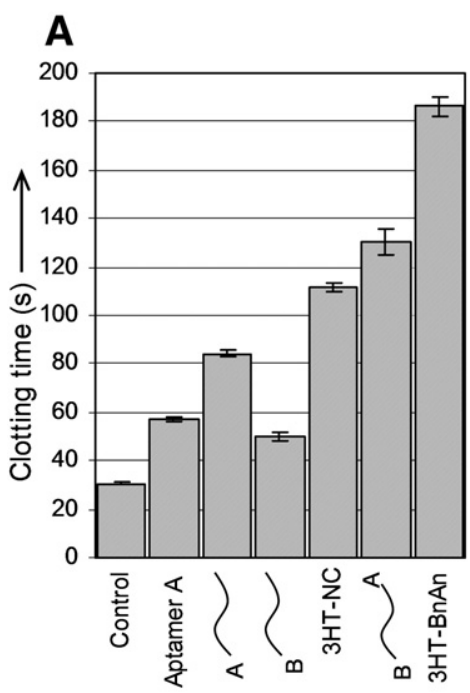

Linker effect.

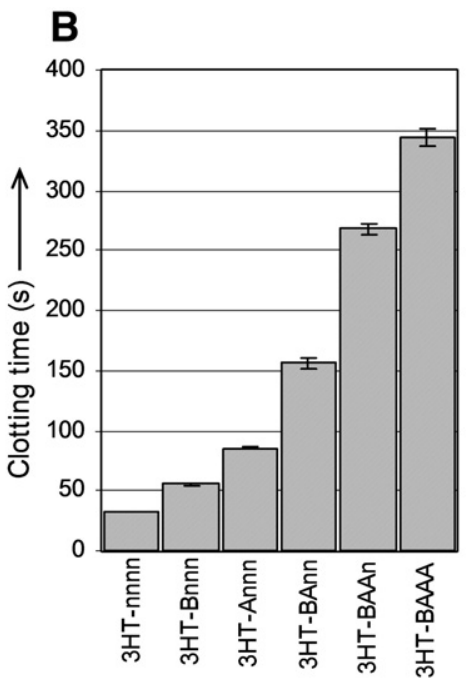

Normalized tile concentration $(1 \mu \mathrm{M})$.

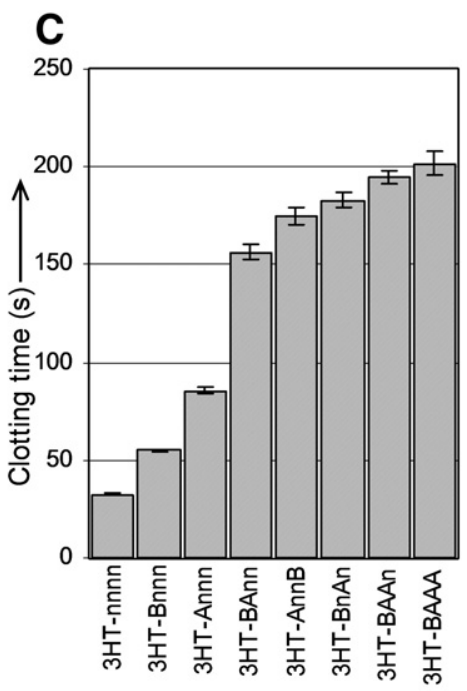

Normalized Aptamer A concentration $(1 \mu \mathrm{M})$.

Figure 2. (A) Significant increase in clotting time is observed when Aptamer B is linked to Aptamer A via single-stranded linker. It increases further when the flexible linker is replaced by relatively more rigid tile (3HT-BnAn). Aptamer A and Aptamer B are at $1 \mu \mathrm{M}$ each. (B) and (C) Anticoagulation increases drastically when Aptamer A and Aptamer B are both present on the tile, as compared to when only Aptamer A is present. (B) Increasing number of Aptamer A per tile significantly increases clotting time for same tile concentration $(1 \mu \mathrm{M})$. (C) However, increasing local concentration of Aptamer A shows a less marked effect on anti-thrombin activity as long as the total aptamer concentration in the solution remains the same $(1 \mu \mathrm{M})$. Clotting time is expressed as mean \pm SEM.

\section{Results}

Comparison of anticoagulant activity of the weave-tile with ssDNA

To determine the effectiveness of the weave tile as a linker compared with ssDNA, the anticoagulant potential of 3HTBnAn was compared with a variety of aptamer-ssDNA constructs using aPTT coagulation assay. The assay revealed that the addition of a DNA tail to Aptamer A led to higher clotting time in comparison with free-floating aptamer (Figure 2, A). Despite possessing weak anticoagulant activity on its own, Aptamer B displayed an additive effect on clotting time when mixed with Aptamer A along with their noncomplementary tails (3HT-NC). In addition, the clotting time showed an increase when the two aptamers were connected via an ssDNA linker. However, by simply adding the complementary strand to form the weave tile, 3HT-BnAn demonstrated significant increase in coagulation time (Figure 2, $A$ ).

\section{Effect of local concentration of Aptamer A on anticoagulation}

After determining the efficiency of the weave tile as a delivery platform, the intent was to maximize the anticoagulant potential of the system by increasing the local concentration of aptamer available for presentation to thrombin. Multiple Aptamer A structures were appended at the corners of the tile to increase the likelihood of re-binding to exosite I in the event of dissociation. Clotting assays conducted with tile concentration normalized to $1 \mu \mathrm{M}$ demonstrated a significant increase in clotting time with addition of each copy of Aptamer A (Figure 2, $B)$. However, to account for the increase in Aptamer A concentration when normalizing the tile concentration, another assay was performed with samples normalized at $1 \mu \mathrm{M}$ for Aptamer A. This assay revealed only modest increases in clotting time between samples with increasing copies of Aptamer A (Figure 2, C).

\section{Optimum tile and optimum configuration}

To identify optimal spacing between Aptamer A and B, the number of helices in the tile and the relative orientation of the two aptamers along the tile were manipulated to provide varying control over inter-aptamer distance. Several weave-tiles were designed from one-helix tiles (1HT) to five-helix tiles (5HT), as illustrated in Figure S2 in the Supplementary Material. Tiles $2 \mathrm{HT}$ to $5 \mathrm{HT}$ were tested in three different configurations whereas 1HT was tested in two, due to the symmetry caused by the single helix. A control tile containing no aptamers and the control sample with no conformation were tested as well. Although a gradual increase in clotting time was observed from $1 \mathrm{HT}$ to $3 \mathrm{HT}$, coagulation time differences were largely indistinguishable among 3HT, 4HT and 5HT configurations (Figure 3). The sole exception was 4HT-BnAn, which demonstrated a sharp peak in coagulation time in comparison with all other structures (Figure 3).

\section{Antidote studies}

After establishing the effectiveness of the tile as a linker, it was vital to demonstrate that the ability to reverse the anticoagulant activity of the aptamers using antidotes was not hindered by linking them to the weave-tile. To determine the most efficient antidote, three short strands complementary to 


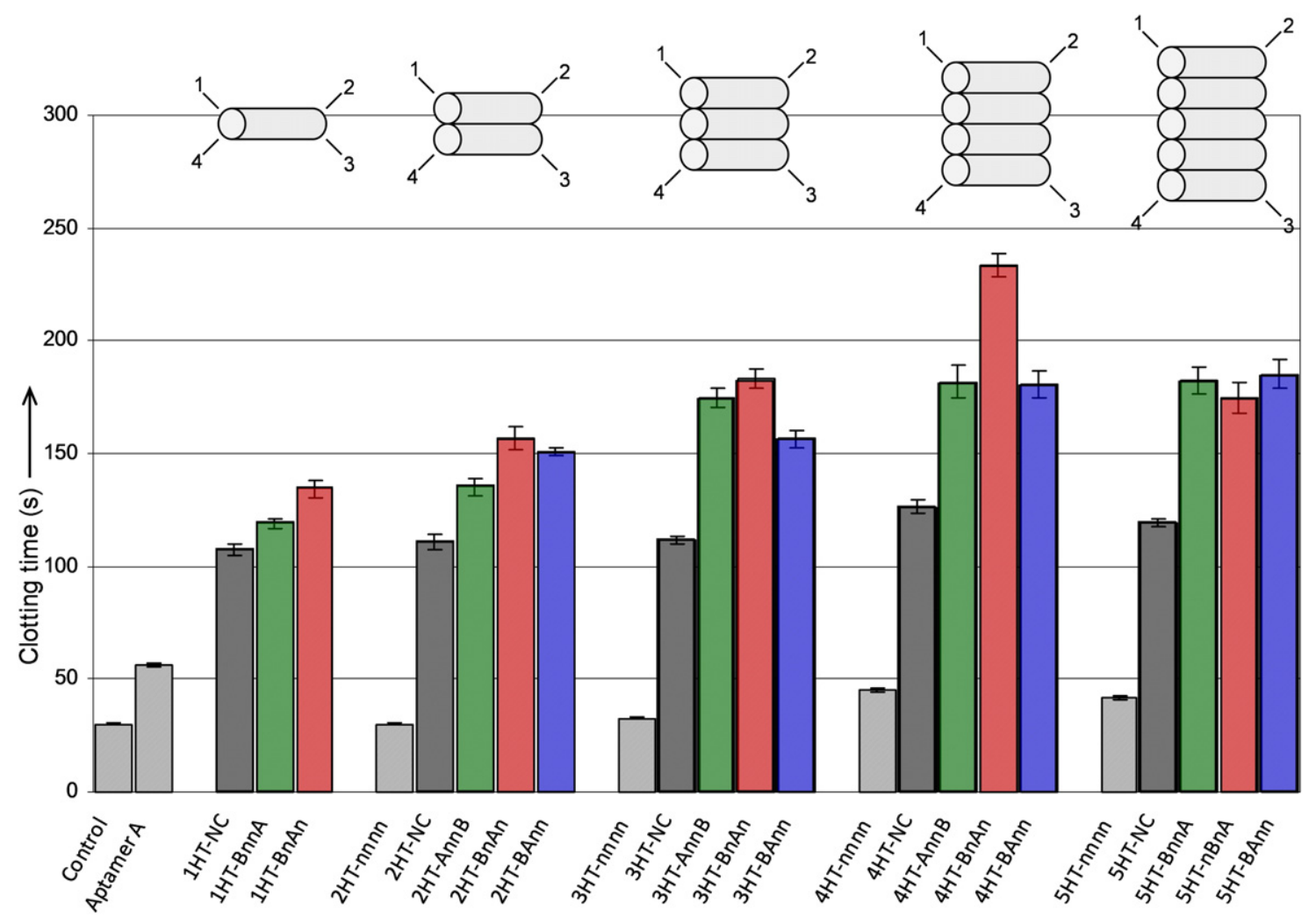

Figure 3. Anticoagulation activity varies depending on the number of helices in the weave tile as well as the configuration of the two aptamers on the tile. Control samples are shown in gray; other samples contain Aptamers A+B either without tile formation (black), in 1+4 configuration (green), in 1+3 configuration (red), or $1+2$ configuration (blue). $4 \mathrm{HT}-\mathrm{BnAn}$ is found to be the optimum tile and optimum configuration. Clotting time is expressed as mean $\pm \mathrm{SEM}$.

various sections of Aptamer A were developed (Figure 4, $A$ ). 2HT-BnAn was used as a representative tile for studying antidote reactivation of thrombin because its constituent strands are shorter and therefore less expensive to use in comparison with larger tiles. Once the parameters like antidote-to-aptamer ratio and time needed for anticoagulation reversal are optimized, antidotes can also be tested and used with the other weave-tiles. Different antidote-to-aptamer ratios were tested to determine what antidote concentration completely reverses the anticoagulant activity of Aptamer A. Figure 4, $B$ shows that residual anticoagulant activity is already greatly reduced at a $1: 1$ antidote-aptamer ratio and decreases to only $5 \%$ residual activity at a 2:1 ratio.

\section{Time-course study}

The degradation of aptamer and antidote efficiency over time was analyzed. Normal aPTT protocol dictates that the DNA samples are incubated for 5 minutes at $37^{\circ} \mathrm{C}$. To observe temporal residual anticoagulant activity progression, time-course studies on free-floating Aptamer A and 2HT-BnAn were conducted over a span of two hours at $37^{\circ} \mathrm{C}$. The results indicate that the anticoagulant activity of Aptamer A decreases rapidly and only about $30 \%$ of the initial activity remains after 2 hours (Figure 4, C). On the contrary, 2HT-BnAn remains much more stable in delaying coagulation and still retains over $70 \%$ of its initial activity after 2 hours. A similarly designed study was conducted to observe the relationship between time and antidote effectiveness. The results demonstrated in Figure 4, $C$ show that all 3 antidotes attached to 2HT-BnAn largely maintained a residual activity level of less than $10 \%$ even after 2 hours. However, antidote bound to free-floating aptamer gradually lost effectiveness over the total time period as Aptamer A regained significant residual anticoagulant activity.

\section{Discussion}

The effect of different molecular contexts on anticoagulant activity was measured using a standard concentration of $1 \mu \mathrm{M}$ Aptamer A (Figure 2, A). The results show that the activity of Aptamer A is increased when an ssDNA tail is attached, in comparison with when Aptamer A free in the solution. Presumably, this is due to electrostatic interactions between the negatively charged tail and the positively charged surface amino acids of thrombin, resulting in higher affinity. This observation confirms a previously reported result. ${ }^{20}$ The clotting time increased by about $23 \%$ when the two aptamers were connected via an ssDNA linker, supporting previously published results. ${ }^{20}$ When the complementary strand is added to the ssDNA linker to form the $3 \mathrm{HT}-\mathrm{BnAn}$ structure, the clotting time increases by another $56 \%$. Thus, increasing the rigidity of the linker over that of ssDNA improves the anticoagulant activity. 

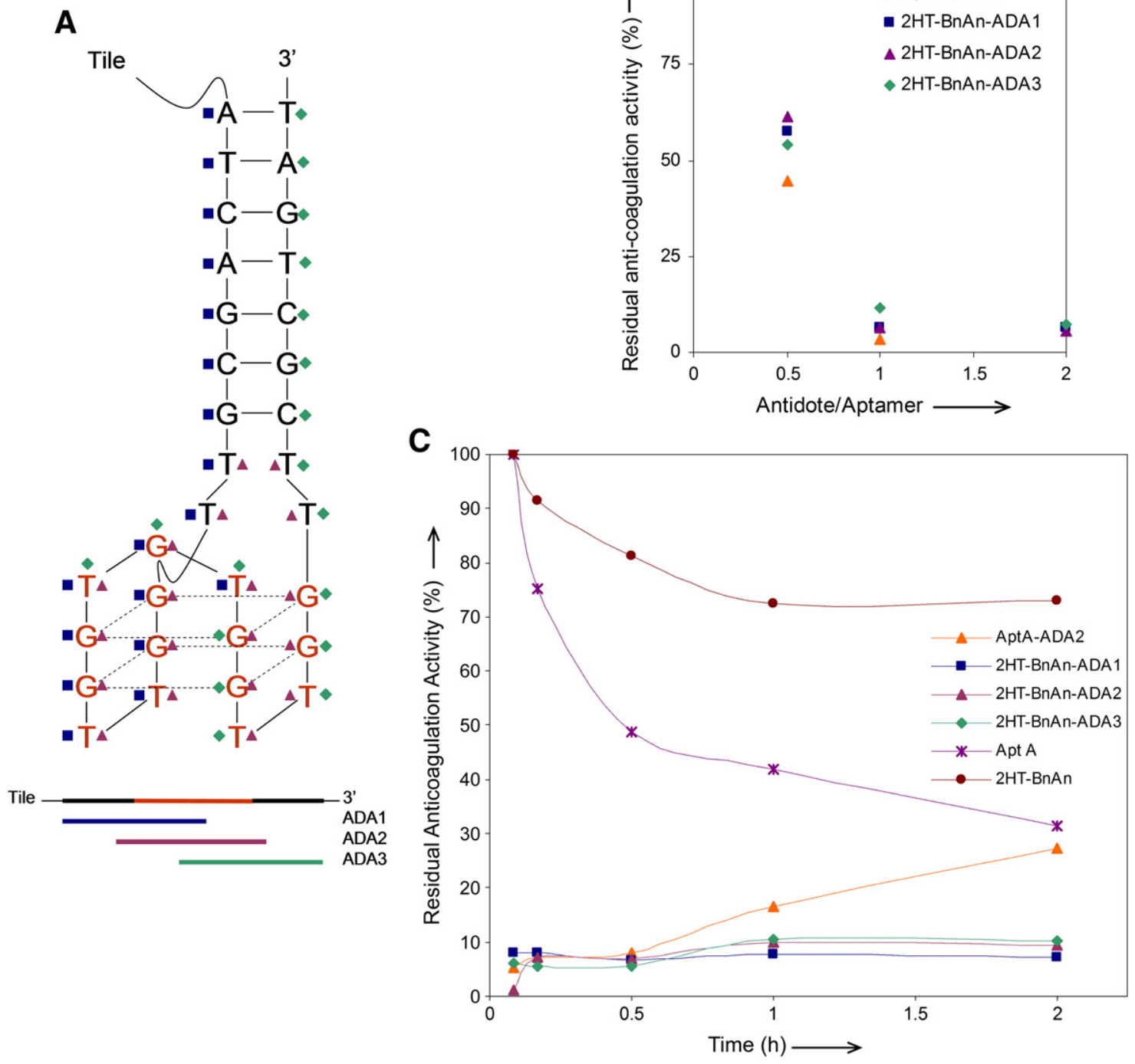

Figure 4. (A) Design of antidotes to reverse the activity of Aptamer A (shown in dark red). The illustration shows three antidotes spanning three regions of the aptamer including the stem region. (B) Reversal of the antithrombin activity of Aptamer A at different antidote to aptamer ratios. 2HT-BnAn is used as a representative tile for comparison with the free Aptamer A in the solution. (C) Stability study of the aptamer activity and its reversal. Free Aptamer A loses its antithrombin activity rapidly, whereas $2 \mathrm{HT}$-BnAn is much more stable with more than $70 \%$ of activity still left after 2 hours. Moreover, reversal of the aptamer activity by antidote (at 2:1 antidote to aptamer ratio) is also much more stable in case of $2 \mathrm{HT}$-BnAn in comparison with free Aptamer A. Error bars are not shown here for the sake of clarity.

It has been established that, for the same amount of Aptamer A in the solution, having higher local concentration of Aptamer A by linking multiple copies increases the clotting time significantly due to cooperativity. ${ }^{11}$ To verify this hypothesis for the two-aptamer system, Aptamer $\mathrm{A}$ was added to the remaining vacant positions on the 3HT-BnAn (see 3HT-BAAn and 3HT-BAAA in Figure 2, B). Tile concentrations were normalized to $1 \mu \mathrm{M}$ over the various samples, and the results demonstrate that having more copies of Aptamer A per tile clearly has considerable impact on anticoagulation. This result could be due to an increase of Aptamer A molecules in the solution. This contention was tested by measuring the anticoagulation activity with normalized $1 \mu \mathrm{M}$ Aptamer A concentration (Figure 2,C). When normalized for Aptamer A concentration, the results still show increased anticoagulant for 3HT-BAAA, but the effect is less pronounced because the sample contained only $333 \mathrm{nM}$ inhibitor (tile-construct) concentration. Additionally, the two aptamers were tried in various configurations for the dual aptamer system, and these choices will be examined further, below. The clotting time increased by $128 \%$ from 3HT-Annn to 3HT-BAnn, which further demonstrates the benefit of Aptamer B on the overall effectiveness of the system due to its high affinity for thrombin.

Because appending additional copies of Aptamer A gave only incremental increases in activity, and because the dual aptamer constructs are less expensive to synthesize, we continued testing using dual aptamer constructs. Our goal was to maximize anticoagulation activity by optimizing the distance between and 
Table 1

Estimated surface distances (ESD, in $\mathrm{nm}$ ) between the points of attachment of the aptamers to the tile for different tiles in various configurations

\begin{tabular}{|c|c|c|c|c|c|}
\hline & $1 \mathrm{HT}$ & $2 \mathrm{HT}$ & $3 \mathrm{HT}$ & $4 \mathrm{HT}$ & 5HT \\
\hline BAnu & 5.1 & 5.1 & 5.1 & 5.1 & 5.1 \\
\hline BnAn & 5.5 & 6.5 & 7.9 & 9.5 & 11.2 \\
\hline \begin{tabular}{|l|} 
BuunA \\
\end{tabular} & 2.0 & 4.0 & 6.0 & 8.0 & 10.0 \\
\hline & $100 x$ & 500 & & 50 & 5 \\
\hline
\end{tabular}

the relative orientation of the two aptamers. Toward this goal, several weave tiles were designed from 1-helix tiles (1HT) to 5-helix tiles (5HT) (Table 1 and Figure S2). Placement of the two aptamers in various configurations over the range of tile sizes provided varying control over inter-aptamer distance. Due to the absence of crossovers, weave tiles are assumed to be nonplanar and are probably curled in the dimension perpendicular to the helix axes (i.e., vertical direction in Figure $1, D$ ). However, because we do not know the extent of curling, we simply used the calculated distance between the points of attachment of the aptamers to the tile on the planar conformation as the estimated surface distance (ESD). The entire range of ESD values is listed in Table 1. Here, it was assumed that the diameter of the DNA double-helix was $2 \mathrm{~nm}$, and the distance between adjacent base pairs along the helical axis was $0.34 \mathrm{~nm} .{ }^{29}$ As can be seen from Table 1, the ESD range available was from $2.0 \mathrm{~nm}$ to $11.2 \mathrm{~nm}$. When the coagulation assay was performed for all the systems (Figure 3), it was found that there was not a significant difference between the clotting times for $3 \mathrm{HT}, 4 \mathrm{HT}$, and $5 \mathrm{HT}$, with the exception of 4HT-BnAn. This particular configuration on the 4HT had the best clotting time by a considerable margin. This shows not only that the distance between the two aptamers is optimum $(\mathrm{ESD}=9.5 \mathrm{~nm})$, but also that they are placed on the tile in the optimum configuration. A previous study using rigid DNA tiles demonstrated an optimal inter-aptamer spacing within the range of 5.3 to $6.9 \mathrm{~nm}$ when monitoring thrombin binding, but anticoagulant activity was not measured. ${ }^{14}$

When the data from Table 1 and Figure 3 were combined and plotted (Figure 5), some very useful insights could be gained. First, in the BAnn configurations (blue bars in Figure 3) the aptamers are separated by 16 base-pairs of dsDNA $(\sim 5 \mathrm{~nm})$ and the tiles are stiff along the axis of the helix due to the DNA persistence length $(\sim 50 \mathrm{~nm})$ being much higher than that. ${ }^{30}$ Aptamer-to-aptamer distance is expected to remain constant irrespective of the number of helices in the tile. Nonetheless, the clotting time steadily increases with tile size (Figures 3 and 5). Hence, the increase in clotting time must be attributed to the increase in the size of the tile. Bigger tiles would have greater van der Waals interactions between the DNA atoms and surface atoms of thrombin. Moreover, there would be a greater electrostatic force of attraction between negatively charged DNA and positively charged surface residues of thrombin. These two factors would effectively combine, thereby giving rise to

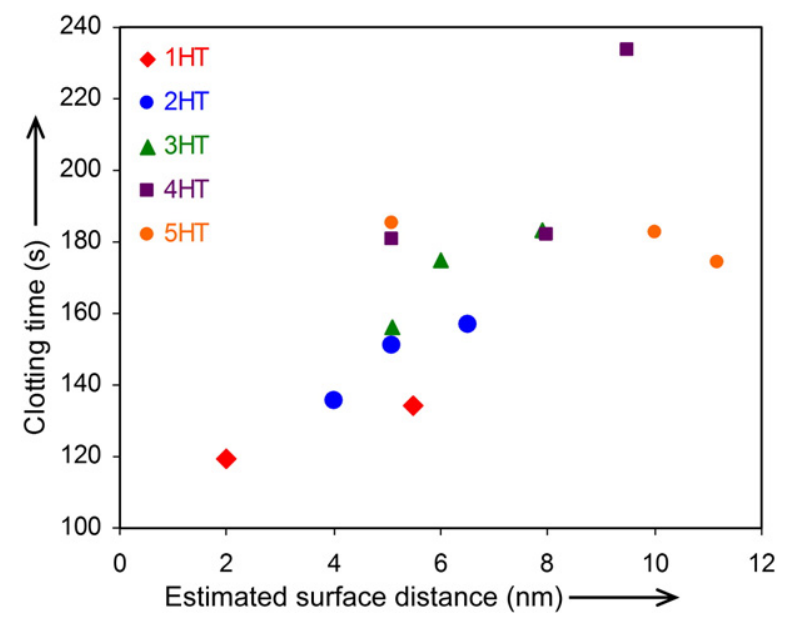

Figure 5. Plot of Anticoagulation Activity vs Estimated Surface Distance (ESD). The anticoagulation activity is a function of the ESD between the two aptamers as well as the configuration of aptamers on the tiles. It also depends on the type of tile used. The error bars are same as in Figure 3 and are not shown here for the sake of clarity.

higher affinity for the larger tiles. Another interesting inference that can be drawn from the plot is that although there is a slight difference between the inter-aptamer distance in the case of 4HTBnAn and 5HT-BnnA, the difference between their respective clotting times is significant. This shows that the choice of tile as well as the aptamer configuration on the tile is as important as the inter-aptamer distance. The choice of the tile and the configuration of the aptamers determine the degree of conformational freedom of the aptamers and hence play a crucial role in presenting the aptamers so that they bind in the appropriate orientation. For the dual aptamer systems tested here, the 4HTBnAn configuration provided the maximum anticoagulant activity and likely represents the best balance of conformational freedom and constraint, such that enthalpic and entropic contributions are balanced to maximize the free energy of association between the DNA nanostructure and thrombin.

As stated earlier, one of the advantages of using DNA aptamers as inhibitors is that they themselves can be functionally modulated by using short complementary oligonucleotide strands as antidotes, thereby providing tight control over aptamer activity. In this study, three DNA antidotes complementary to three different regions of Aptamer A were designed and tested (Figure 4, A). Different antidote-to-aptamer ratios were tested using $2 \mathrm{HT}-\mathrm{BnAn}$ as the representative tile to determine what antidote concentration completely reverses the anticoagulant activity of Aptamer A. Figure S4 in the Supplementary Material illustrates that inactivation of aptamer should be nearly complete at $1: 1$ ratio with some unbound native aptamer still left in solution, whereas at a 2:1 antidote to aptamer ratio, inactivation of aptamer should be complete for all three antidotes. This is verified in coagulation assays of the same (Figure $4, B$ ). There is only about $5 \%$ residual activity after 5 minutes of incubation for 2:1 antidote to aptamer ratio at $37^{\circ} \mathrm{C}$. The formula for calculation of residual activity has been mentioned earlier and the PAGE results showing antidote/aptamer interaction are included in the Supplementary Material (Figure S4 and S5). 
In all the experiments reported here, the various aptamer systems were incubated with the plasma for 5 minutes before triggering the coagulation cascade. Moreover, the antidote experiments also involved a further incubation of only 5 minutes. This shows that antithrombin activity of the dual aptamer system is very rapid, as is its reversal. This finding is an important consideration for therapeutic applications since the aptamer anticoagulant could be rapidly controlled in clinical settings after the completion of surgery if bleeding complications arise. Another key factor to be ascertained is the stability and durability of the system over a longer period. It is vital to determine how long the aptamer system maintains its anticoagulation potential and how effective the antidotes are in keeping it reversed over that period. To determine this, timecourse studies were performed in plasma over 2 at $37^{\circ} \mathrm{C}$. The results indicate that the residual anticoagulation potential of Aptamer A is significantly less than that of 2HT-BnAn (Figure 4, C). It can be inferred that placing Aptamer A on a weave-tile platform increases its stability. Similarly, the reversal of free Aptamer A using antidote is not stable over 2 hours (Figure 4, C). On the other hand, the reversal of the activity of $2 \mathrm{HT}$ BnAn by various antidotes is very stable and the residual activity is less than $10 \%$ even after 2 hours. The loss of activity of aptamers may be attributed to the degradation due to the presence of nucleases in plasma. By incorporating appropriate chemical modifications, the degradation can be minimized and hence it is possible to achieve a considerably more stable system.

In summary, we have successfully demonstrated the application of DNA weave-tiles in reliable nano-positioning of multiple thrombin-binding aptamers to create a functional DNA nanostructure. These aptamer-tile systems have then been used as anticoagulants in blood plasma clotting experiments. It was established that these tiles act as a much better platform connecting the two aptamers in comparison with a flexible single-stranded polynucleotide linker. During the process, an optimum two-aptamer complex with an optimum configuration of aptamers on it (4HT-BnAn) was engineered that has significantly higher anticoagulation activity in omparison with other tiles and other configurations. It is imperative to note here that such a system has to be selected via screening of a variety of different tiles with different aptamer arrangements and it cannot be designed by taking pre-existing parameters into consideration. It is evident from the our study that the distance between the two aptamers, their relative configuration on the tile, and the type of tile being used are all crucial factors. The anticoagulation effect of the system is rapid and quite stable. Furthermore, it is possible to conveniently, rapidly, and stably reverse the anticoagulation by adding an antidote strand. Efficient reversal of activity by antidotes gives aptamers a distinct advantage over other therapeutic antagonists such as antibodies, peptides, or other smaller molecules. ${ }^{22}$ The study represents a major step toward realizing the application of DNA nanotechnology in therapeutics. It is proposed that the current strategy should be extended to target other key components of the coagulation cascade with suitable choice of aptamers with the goal of engineering the most efficient system for anticoagulation. Moreover, it is recommended that the system presented here be tested in animal models as the first step towards clinical trials.

\section{Appendix A. Supplementary data}

Supplementary data to this article can be found online at doi:10.1016/j.nano.2011.08.011.

\section{References}

1. Fu T, Seeman NC. DNA double-crossover molecules. Biochemistry 1993;32:3211-20.

2. LaBean TH, Yan H, Kopatsch J, Liu F, Winfree E, Reif JH, et al. Construction, analysis, ligation, and self-assembly of DNA triple crossover complexes. J Am Chem Soc 2000;122:1848-60.

3. Yan H, Park SH, Finkelstein G, Reif JH, LaBean TH. DNA-templated self-assembly of protein arrays and highly conductive nanowires. Science 2003;301:1882-4.

4. Liu D, Wang M, Deng Z, Walulu R, Mao C. Tensegrity: Construction of rigid DNA triangles with flexible four-arm DNA junctions. J Am Chem Soc 2004; $126: 2324-5$.

5. Mathieu F, Liao S, Kopatsch J, Wang T, Mao C, Seeman NC. Six-helix bundles designed from DNA. Nano Lett 2005;5:661-5.

6. Rothemund PWK. Folding DNA to create nanoscale shapes and patterns. Nature 2006;440:297-302.

7. Douglas SM, Dietz H, Liedl T, Högberg B, Graf F, Shih WM. Selfassembly of DNA into nanoscale three-dimensional shapes. Nature 2009;459:414-8.

8. Andersen ES, Dong M, Nielsen MM, Jahn K, Subramani R, Mamdouh W, et al. Self-assembly of a nanoscale DNA box with a controllable lid. Nature 2009;459:73-6.

9. Winfree E, Liu F, Wenzler LA, Seeman NC. Design and self-assembly of two-dimensional DNA crystals. Nature 1998;394:539-44.

10. He Y, Ye T, Su M, Zhang C, Ribbe AE, Jiang W, et al. Hierarchical self-assembly of DNA into symmetric supramolecular polyhedra. Nature 2008;452:198-201.

11. Hansen MN, Zhang AM, Rangnekar A, Bompiani KM, Carter JD, Gothelf KV, et al. Weave tile architecture construction strategy for DNA nanotechnology. J Am Chem Soc 2010;132:14481-6.

12. Shen Z, Yan H, Wang T, Seeman NC, Paranemic crossover DNA. A generalized Holliday structure with applications in nanotechnology. J Am Chem Soc 2004;126:1666-74.

13. Zhang J, Liu Y, Ke Y, Yan H. Periodic square-like gold nanoparticle arrays templated by self-assembled 2D DNA nanogrids on a surface. Nano Letters 2006;6:248-51.

14. Rinker S, Ke Y, Liu Y, Chhabra R, Yan H. Self-assembled DNA nanostructures for distance-dependent multivalent ligand-protein binding. Nat Nanotechnology 2008;3:418-22.

15. Ellington AD, Szostak JW. In vitro selection of RNA molecules that bind specific ligands. Nature 1990;346:818-22.

16. Tuerk C, Gold L. Systematic evolution of ligands by exponential enrichment: RNA ligands to bacteriophage T4 DNA polymerase. Science 1990;249:505-10.

17. Bock LC, Griffin LC, Latham JA, Vermaas EH, Toole JJ. Selection of single-stranded DNA molecules that bind and inhibit human thrombin. Nature 1992;355:564-6.

18. Di Cera E. Thrombin interactions. Chest 2003;124:11S-7S.

19. Kwaan HC, Samama MM. Anticoagulant drugs: An update. Expert Rev Cardiovasc Ther 2004;2:511-22.

20. Müller J, Wulffen B, Pötzch B, Mayer G. Multidomain targeting generates a high-affinity thrombin-inhibiting bivalent aptamer. ChemBioChem 2007;8:2223-6.

21. White RR, Sullenger BA, Rusconi CP. Developing aptamers into therapeutics. J Clin Invest 2000;106:929-34.

22. Rusconi CP, Scardino E, Layzer J, Pitoc GA, Ortel TL, Monroe D, et al. RNA aptamers as reversible antagonists of coagulation factor IXa. Nature 2002;419:90-4.

23. Dyke CK, Steinhubl SR, Kleiman NS, Cannon RO, Aberle LG, Lin M, et al. First-in-human experience of an antidote-controlled anticoagulant 
using RNA aptamer technology: A phase 1a pharmacodynamic evaluation of a drug-antidote pair for controlled regulation of Factor IXa activity. Circulation 2006;114:2490-7.

24. Chan MY, Rusconi CP, Alexander JH, Tonkens RM, Harrington RA, Becker RC. A randomized, repeat-dose, pharmacodynamic and safety study of an antidote-controlled factor IXa inhibitor. J Thromb Haemost 2008;6:789-96.

25. Chan MY, Cohen MG, Dyke CK, Myles SK, Aberle LG, Lin M, et al. Phase $1 \mathrm{~b}$ randomized study of antidote-controlled modulation of factor IXa activity in patients with stable coronary artery disease. Circulation 2008;117:2865-74.

26. Cohen MG, Purdy DA, Rossi JS, Grinfeld LR, Myles SK, Aberle LH, et al. First clinical application of an actively reversible direct factor IXa inhibitor as an anticoagulation strategy in patients undergoing percutaneous coronary intervention. Circulation 2010;122:614-22.

27. Padmanabhan K, Tulinsky A. An ambiguous structure of a DNA 15-mer thrombin complex. Acta Crysta 1996;D52:272-82.

28. Mammen M, Choi SK, Whitesides GM. Polyvalent interactions in biological systems: Implications for design and use of multivalent ligands and inhibitors. Angew Chem Int Ed 1998;37: 2755-94.

29. Watson JD, Crick FHC. A Structure for Deoxyribose Nucleic Acid. Nature 1953;171:737-8.

30. Barkley MD, Zimm BH. Theory of twisting and bending of chain macromolecules - Analysis of the fluorescence depolarization of DNA. J Chem Phys 1979;70:2991-3007. 